\title{
S-shaped wound closure following the excision of 2 adjacent pigmented nevi
}

\author{
Yueqian Zhu ${ }^{1 \#}$, Xiuxia Wang ${ }^{2 *}$, Shujun Wang ${ }^{3}$, Naihui Zhou ${ }^{1}$, Ke Liu ${ }^{4}$ \\ ${ }^{1}$ Department of Dermatology, The First Affiliated Hospital of Soochow University, Suzhou, China; ${ }^{2}$ Department of Plastic and Reconstructive \\ Surgery, Shanghai Ninth People's Hospital, Shanghai Jiao Tong University School of Medicine, Shanghai, China; ${ }^{3}$ Department of Burns and Plastic \\ Surgery, Yancheng No. 1 People's Hospital, Yancheng, China; ${ }^{4}$ Department of Dermatology, Shanghai Ninth People's Hospital, Shanghai Jiao Tong \\ University School of Medicine, Shanghai, China \\ Contributions: (I) Conception and design: Y Zhu; (II) Administrative support: N Zhou; (III) Provision of study materials or patients: K Liu; (IV) \\ Collection and assembly of data: X Wang; (V) Data analysis and interpretation: S Wang; (VI) Manuscript writing: All authors; (VII) Final approval of \\ manuscript: All authors. \\ \#These authors contributed equally to this work. \\ Correspondence to: Ke Liu. Department of Dermatology, Shanghai Ninth People’s Hospital, Shanghai Jiao Tong University School of Medicine, 639 \\ Zhizaoju Road, Shanghai 200011, China. Email: qq593783531@163.com; Naihui Zhou. Department of Dermatology, The First Affiliated Hospital \\ of Soochow University, 188 Shizi Road, Suzhou 215000, China. Email: zhounaihui@163.com.
}

Background: The main goal of excision of a pigmented nevus is to achieve an esthetically pleasing result. A single nevus can be removed by simple excision, whereas S-shaped suture can be used for the excision of 2 adjacent nevi. However, the choice of suturing method is based on the experience of the dermatologic surgeon, as there is no uniform standard for suture following the excision of 2 adjacent nevi. The aim of the present study was to determine whether S-shaped wound closure is appropriate for the excision of 2 adjacent nevi.

Methods: The outcomes of 21 patients who underwent simultaneous resection of 2 adjacent nevi were retrospectively reviewed. Of these patients, 17 chose S-shaped suture and 4 chose direct suture. Patients were followed-up for more than 6 months to review their postoperative scars. Differences between the 2 methods were compared based on the patient and observer scar assessment scale. Diameters and the proportional relationship between the 2 nevi were analyzed.

Results: Patients who underwent S-shaped wound closure surgery were more satisfied compared to those who had direct suturing $(\mathrm{P}<0.05)$; the nevus diameter-to-spacing diameter ratio was $0.68 \pm 0.35: 1: 0.99 \pm 0.56$ in the S-shaped wound closure group.

Conclusions: S-shaped wound closure following the excision of 2 adjacent nevi resulted in better patient satisfaction than the conventional direct suturing method.

Keywords: S-shaped wound closure; pigmented nevus; excision; suturing; adjacent nevi

Submitted Oct 09, 2020. Accepted for publication Dec 04, 2020.

doi: $10.21037 /$ atm-20-7167

View this article at: http://dx.doi.org/10.21037/atm-20-7167

\section{Introduction}

A pigmented nevus is the most common benign tumor of the skin. Patients who request surgical resection often require not only a clean resection, but also more attention to postoperative esthetics; therefore, the dermatologic surgeon must choose the most appropriate techniques and procedures. Surgical removal of a single pigmented nevus is relatively simple, and a satisfactory outcome is often achieved. Although removal of 2 adjacent pigmented nevi is more challenging, there is a variety of surgical options.

Most lesions are nearly round. From a geometric point 


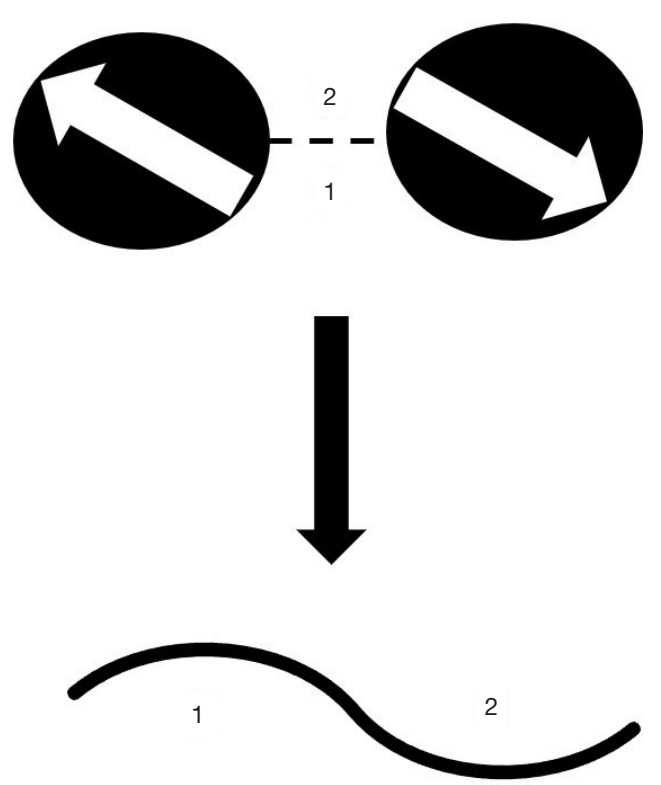

Figure $1 \mathrm{~S}$-shaped wound technique model. Two adjacent nevi are cut along the edges and the normal skin in the middle is used to fill in the cuts to form an $\mathrm{S}$ shape.

of view, after the removal of 2 nevi along the boundary, if both are directly pulled into a straight line without trimming the "dog ear" that is formed during wound closure, then the length of this straight line should be half of the circumference of the skin lesion (i.e., $1 / 2 \pi \mathrm{d}$, where $\mathrm{d}$ is the diameter of the lesion). However, if the distance between the centers of the 2 nevi is $>1 / 4 \pi(d 1+d 2)$, then both are considered to exist independently of one another and the surgery can be performed as though there was a single pigmented nevus, and each can be removed along the skin line. When 2 nevi are close to each other, they are treated as the same mass and only a fusiform incision is created to achieve a relatively satisfactory result. As reported in the literature, when there is appropriate distance between the 2 nevi, a S-shaped suturing method might be a good choice (1).

The S-shaped wound closure method was first reported for the resection of dumbbell-shaped keloids (2). In this model, 2 adjacent nevi are cut along the edges, and the normal skin in the middle is used to fill in the cuts, while leaving the defects; finally, an S-shaped wound is formed. This option results in greater satisfaction for both the surgeon and patient.

However, dermatologic surgeons usually rely upon their own experience to select the most appropriate surgical method, as there is no quantitative standard. However, as there is no consensus on an appropriate diameter and the proportional relationship between the 2 nevi, it is important to determine the most suitable distance between the nevi for S-shaped sutures.

In the present study, the postoperative recovery of 21 patients who had 2 nevi removed simultaneously were retrospectively analyzed to determine an appropriate distance between 2 nevi for the application of S-shaped sutures.

We present the following article in accordance with the STROBE reporting checklist (available at http://dx.doi. org/10.21037/atm-20-7167).

\section{Methods}

The study was conducted in accordance with the Declaration of Helsinki (as revised in 2013). The study was approved by Shanghai Ninth People's Hospital, Shanghai Jiao Tong University School of Medicine, ethics board of SH9H-2020-T85-1and informed consent was taken from all individual participants.

\section{Patients and methods}

From January 2017 to December 2017, preoperative and postoperative follow-up photos of 21 patients with 2 adjacent nevi were retrospectively reviewed, and patient satisfaction was investigated. The choice of surgical method was based on previous reports, geometric calculations, estimates of the diameter, and distance of the pigmented nevi to determine the most suitable situation for the implementation of S-shaped wound closure. The distance between the 2 nevi ranged from 0 to $1 / 4 \pi(d 1+d 2)$. However, when informed of the surgical procedure, 4 patients refused S-shaped wound closure because of the curved scars. Therefore, S-shaped wound closure was applied in only 17 cases (Figure 1). Four patients who insisted on straight-line slitting or slitting alone were used as the control group. A 5-0 polydioxanone synthetic absorbable suture (PDS II; ETHICON, USA) thread was used for intradermal closure, while a 6-0 Prolene thread (ETHICON, USA) was used for external closure. Silicone patches and tension-reducing tape were applied externally after removal of the thread. Patients were followed-up for more than 6 months. All procedures were performed by the same surgeons ( $\mathrm{KL}$ and $\mathrm{YZ}$ ) in the same operating room of the Ninth People's Hospital, Shanghai Jiaotong University (Shanghai, China). Silicon patches and tension-reducing tape are used to reduce scar formation after remove the 
Table 1 Patient characteristics of the S-shape wound closure group

\begin{tabular}{|c|c|c|c|c|c|}
\hline Patient No. & Patient age (years) & Sex & Postoperative follow-up (months) & Lesion location & Ratio (diameter/distance/diameter) \\
\hline 3 & 36 & Female & 14 & $\begin{array}{l}\text { Left nasolabial } \\
\text { groove }\end{array}$ & $0.75: 1: 0.75$ \\
\hline 5 & 38 & Female & 15 & $\begin{array}{l}\text { Left nasolabial } \\
\text { groove }\end{array}$ & $1.2: 1: 1.4$ \\
\hline 6 & 56 & Female & 8 & Nose & $0.75: 1: 0.75$ \\
\hline 7 & 38 & Female & 9 & $\begin{array}{l}\text { Left nasolabial } \\
\text { groove }\end{array}$ & $0.6: 1: 0.6$ \\
\hline 10 & 40 & Female & 14 & Upper lip & $0.33: 1: 0.33$ \\
\hline 11 & 17 & Male & 10 & Scalp & $0.44: 1: 0.67$ \\
\hline 12 & 34 & Male & 14 & Scalp & $0.63: 1: 2.25$ \\
\hline 13 & 52 & Female & 6 & Chest & $0.43: 1: 0.57$ \\
\hline 14 & 29 & Male & 12 & Right cheek & $1: 1: 1.25$ \\
\hline
\end{tabular}

stitches.

The patient and observer scar assessment scale (POSAS) were used to score the postoperative wound. The POSAS consists of 2 parts: the observer scale and the patient scale. The observer scale is based on 6 items (vascularity, pigmentation, thickness, relief, pliability, surface area), with scores ranging from 6 to 60 points (3). The patient scale is also based on 6 items (pain, itching, color, stiffness, thickness, surface irregularity), with scores ranging from 6 to 60 points. Data of the control and experimental groups were compared to identify statistically significant differences. Cases with a POSAS score of $>10$ points were selected for careful measurement of the diameter of each lesion ( $\mathrm{d} 1$ and $\mathrm{d} 2)$ and the distance between lesions $\mathrm{d} 1$ and $\mathrm{d} 2$.

All statistical analyses were performed using SPSS Statistics for Windows, version 17.0 (SPSS, Chicago, IL, USA). Student's $t$-test was used to identify significant differences between groups. $\mathrm{P}<0.05$ indicated statistical significance.

\section{Results}

Three patients were lost to follow-up; therefore, the data of 18 patients were included for analysis. The S-shape group consisted of 14 patients (6 males and 8 females). The average age of all patients was 36 years. 7 were located on the face, 2 on the scalp, and 1 on the chest (Table 1). Representative preoperative and postoperative photos of the S-shape group are shown in Figure 2. The control group consisted of 4 female patients; 3 were located on the face, 1 was located on the neck (Table 2).

\section{Satisfaction comparison between the experimental and control groups}

There were significant differences in the observer scale and patient scale scores between the experimental and control groups (6.78 and $6.21 v s .13 .5$ and 14.5, respectively, $\mathrm{P}<0.05$ ) (Table 3), indicating that patients were more satisfied with postoperative scaring and the overall esthetic effect of S-shaped wound closure.

\section{Relationship between the 2 skin lesions}

In the S-shape wound closure group, the scores of the observer and patient scales were all $<10$ points. Therefore, all patients in the S-shape wound closure group were 

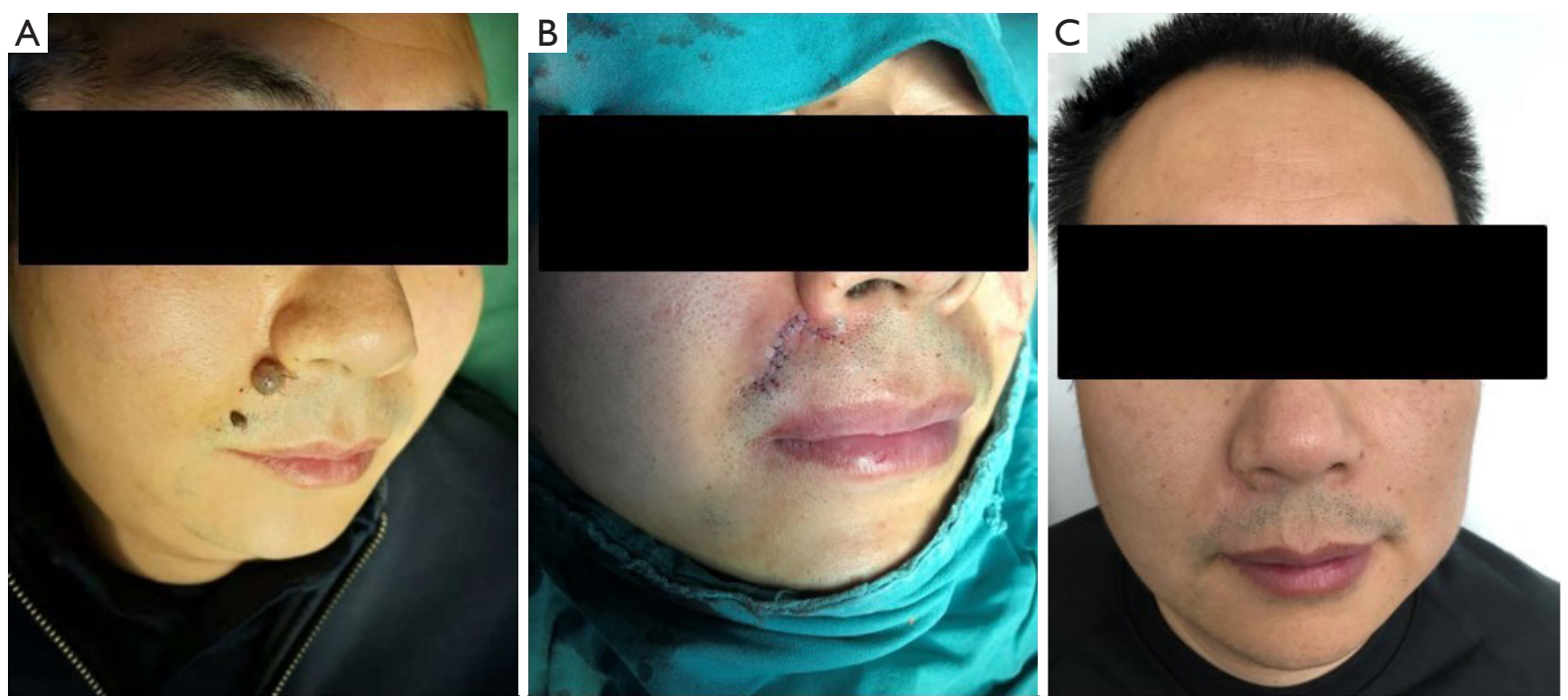

Figure 2 Treatment of 2 adjacent nevi using S-shaped wound closure technique. (A) Preoperative view; 2 nevi located in the right nasolabial groove. (B) Immediate postoperative view; S-shaped wound closure was formed. (C) Postoperative view after 12 months; the scar was not obvious.

Table 2 Patient characteristics of the control group

\begin{tabular}{llllll}
\hline Patient No. & Patient age (years) & Sex & Postoperative follow-up (months) & Lesion location Ratio (diameter/distance/diameter) \\
\hline 1 & 41 & Female & 12 & neck & $0.56: 1: 0.66$ \\
2 & 20 & Female & 11 & nose & $3: 1: 3$ \\
3 & 22 & Female & 10 & Right cheek & $2: 1: 6$ \\
4 & 21 & Female & 12 & Left cheek & $0.4: 1: 0.5$ \\
\hline
\end{tabular}

Table 3 Patient and observer scar assessment scale and ratio relationship between S-shape and non-S-shape group

\begin{tabular}{llll}
\hline Group & Observer scale & Patient scale & Ratio (diameter/distance/diameter) \\
\hline S-shape & $6.78 \pm 0.80$ & $6.21 \pm 0.43$ & $0.68 \pm 0.35: 1: 0.99 \pm 0.56$ \\
None S-shape & $13.50 \pm 3.79$ & $14.5 \pm 2.08$ & $1.49 \pm 1.07: 1: 2.54 \pm 2.23$ \\
P value & 0.037 & 0.004 & \\
\hline
\end{tabular}

$\mathrm{P}<0.05$ indicates statistical significance.

included for the analysis of outcomes of 2 adjacent nevi. The distance between the 2 lesions ( 11 and $\mathrm{d} 2$ ) was set to a value of 1 ; therefore, the $\mathrm{d} 1: \mathrm{D}: \mathrm{d} 2$ ratio was $0.68 \pm 0.35: 1: 0.99 \pm 0.56$. In the control group, the $\mathrm{d} 1: \mathrm{D}: \mathrm{d} 2$ ratio was $1.49 \pm 1.07: 1: 2.54 \pm 2.23$ (Table 3).

\section{Discussion}

Nevus excision is the most common surgery in the dermatologic field. Generally, the classic fusiform resection method is applied for a single nevus, while fractional resection or the creation of flaps is applied for large nevi $(4,5)$. At present, there is no consensus on the best surgical method for the removal of 2 adjacent nevi. In the present study, excision was performed along the periphery of the edges of the 2 nevi while maintaining a 1-mm safety margin; this formed 2 adjacent notches on the skin. The skin between both notches were resected and the flap was used 
to cover the defect, which created an S shape. This method resulted in good patient satisfaction.

The dermatologic surgeon's concern is the loss of normal skin, as well as esthetic appearance. A direct fusiform incision often involves the removal of the normal skin between the 2 skin lesions. The most obvious advantage of S-shaped wound closure is that the normal skin can be used to fill the defects after removal of the lesions, thereby reducing the loss of normal skin. Second, skin modification greatly reduces and changes the direction of skin tension, without causing extension of the incision. Third, the resulting "dog ear" formations are smaller compared with direct slits. Fourth, in the facial area, the W-reform concept can be adopted according to the dermatoglyph in order to adapt the partial tangent of the S-shaped incision to conform to the dermatoglyph. This reduces scar visibility and enhances the cosmetic effect (6). To achieve a better esthetic effect, silicone patches and tension-reducing tape are still required postoperatively (7).

Of the 14 cases of pigmented nevi, 11 were on the face, 2 on the scalp, and 1 on the chest. All cases achieved good results, suggesting a wide range of applicable sites for S-shaped wound closure. In the present study, the ratio of the nevus diameter, spacing, and the other nevus diameter among patients who were satisfied was $0.68 \pm 0.35: 1: 0.99 \pm 0.56$. In clinical practice, the dermatologist often encounters patients with 2 lesions that are vastly different in size. The choice of S-flap is equally applicable to 2 nevi with different diameters.

Because the wound length of the S-flap is not comparable to that of a straight incision, some patients may be concerned that an S-shaped incision will result in a longer incision line. For nevi on the face, an S-shaped flap can mimic the shape of a $W$ to achieve a curved shape, which can be used to lighten the scar, so that even if the wound is visible, scaring is less noticeable. Therefore, for such cases, increasing the length of the scar makes sense. Lesions on the back, chest, and other areas that are prone to scar hyperplasia require careful selection of the S-flap.

For more than 2 consecutive nevi, the S-flap method is still applicable if there is normal skin among the lesions. In the present study, the ratio of the nevus diameter, spacing, and nevus diameter was $0.68 \pm 0.35: 1: 0.99 \pm 0.56$, suggesting that the proposed method is not only applicable for the removal of pigmented nevi, but also other benign skin rashes and scars. However, future clinical studies are needed to confirm these results.

A limitation of the present study was the limited number of patients, which may have biased the results. Furthermore, the simultaneous removal of 2 adjacent pigmented nevi is not common. Nonetheless, we plan to extend these findings in future studies.

\section{Conclusions}

We reported S-shaped wound closure for adjacent pigmented nevi after excision. This procedure resulted in better patient satisfaction than the conventional direct suturing method.

\section{Acknowledgments}

Funding: The present study was supported by the Fundamental Research Program of the Ninth People's Hospital, Shanghai Jiao Tong University School of Medicine (grant no. JYZZ035); the Interdisciplinary Research Program of the Ninth People's Hospital, Shanghai Jiao Tong University School of Medicine (grant no. YG2019QNA13); and the National Natural Science Foundation of China (grant no. 82001978, 81703144). Natural Science Foundation of Jiangsu Province (BK20160350).

\section{Footnote}

Reporting Checklist: The authors have completed the STROBE reporting checklist. Available at http://dx.doi. org/10.21037/atm-20-7167

Data Sharing Statement: Available at http://dx.doi. org/10.21037/atm-20-7167

Conflicts of Interest: All authors have completed the ICMJE uniform disclosure form (available at http://dx.doi. org/10.21037/atm-20-7167). The authors have no conflicts of interest to declare.

Ethical Statement: The authors are accountable for all aspects of the work in ensuring that questions related to the accuracy or integrity of any part of the work are appropriately investigated and resolved. The study was conducted in accordance with the Declaration of Helsinki (as revised in 2013). The study was approved by Shanghai Ninth People's Hospital, Shanghai Jiao Tong University School of Medicine, ethics board of SH9H-2020-T85-1 and informed consent was taken from all individual participants. 
Open Access Statement: This is an Open Access article distributed in accordance with the Creative Commons Attribution-NonCommercial-NoDerivs 4.0 International License (CC BY-NC-ND 4.0), which permits the noncommercial replication and distribution of the article with the strict proviso that no changes or edits are made and the original work is properly cited (including links to both the formal publication through the relevant DOI and the license). See: https://creativecommons.org/licenses/by-nc-nd/4.0/.

\section{References}

1. Karaman K, Ozturk S, Tugmen C, et al. S-shaped wide excision with primary closure for extensive chronic pilonidal sinus disease. Case Rep Surg 2014;2014:451869.

2. Komatsu S, Azumi S, Hayashi Y, et al. S-shaped Wound Closure Technique for Dumbbell-shaped Keloids. Plast Reconstr Surg Glob Open 2017;5:e1278.

Cite this article as: Zhu Y, Wang X, Wang S, Zhou N, Liu K. S-shaped wound closure following the excision of 2 adjacent pigmented nevi. Ann Transl Med 2020;8(24):1648. doi: 10.21037/atm-20-7167
3. Truong PT, Lee JC, Soer B, et al. Reliability and validity testing of the Patient and Observer Scar Assessment Scale in evaluating linear scars after breast cancer surgery. Plast Reconstr Surg 2007;119:487-94.

4. Lim JY, Song WK, Whang KK. Scar-Saving Flap during Serial Excision by Borrowing from the Opposite Side. Ann Dermatol 2008;20:120-5.

5. Kwon KH, Lee DG, Koo SH, et al. Usefulness of v-y advancement flap for defects after skin tumor excision. Arch Plast Surg 2012;39:619-25.

6. Izaguirre H, Navarro C. Rhomboid-to-"W" technique for excision and closure of facial skin lesions. J Maxillofac Surg 1983;11:207-10.

7. Kim S, Choi TH, Liu W, et al. Update on scar management: guidelines for treating Asian patients. Plast Reconstr Surg 2013;132:1580-9.

(English Language Editor: R. Scott) 